\title{
SÍNTESE DE REDES FLEXÍVEIS DE TROCADORES DE CALOR VIA PROGRAMAÇÃO MATEMÁTICA USANDO UM MÉTODO DE OTIMIZAÇÃ̃O SEQUENCIAL
}

\author{
C. B. MIRANDA ${ }^{1}$, M. A. S. S. RAVAGNANI ${ }^{1}$ \\ ${ }^{1}$ Universidade Estadual de Maringá, Departamento de Engenharia Química \\ E-mail para contato: camila.mir4@gmail.com
}

\begin{abstract}
RESUMO - A síntese de redes de trocadores de calor (RTC) é um dos ramos mais importantes para o estudo de processos industriais, pois permite que se reduza o gasto com utilidades. Este trabalho apresenta um método sequencial via programação matemática para a síntese de RTC flexíveis, redes estas que suportam variações nas capacidades térmicas e nas temperaturas das correntes em um número finito de períodos de operação. $\mathrm{O}$ modelo desenvolvido usa a técnica de decomposição em três objetivos: custo mínimo de utilidades para cada período de operação; mínimo número de unidades de troca de calor; e mínimo custo de investimento. $\mathrm{O}$ presente trabalho tem como objetivo alcançar a configuração da rede flexível automaticamente, por meio da formulação de um problema de programação não linear (PNL). Propõe-se uma superestrutura multiperiódica e a criação de dois conjuntos e um parâmetro para reduzir o número de variáveis e de restrições envolvidas nessa formulação matemática. Utilizou-se um exemplo da literatura para testar a aplicabilidade do modelo desenvolvido. Os resultados obtidos comprovaram a viabilidade do modelo, atingindo resultados melhores do que os apresentados na literatura.
\end{abstract}

\section{INTRODUÇÃO}

Redes flexíveis de trocadores de calor são redes capazes de suportar mudanças nas condições de operação em um número finito de períodos de operação, como variações nas temperaturas de entrada e saída e nas vazões das correntes de processo (MÓDENES, 1995). A abordagem do problema de otimização pode trazer resultados mais interessantes do ponto de vista da aplicação industrial, permitindo sintetizar a rede de maneira sistemática, incluir mais de um tipo de utilidade quente ou fria e introduzir restrições quando necessário. Além disso, por meio do método sequencial, divide-se o problema em uma série de subproblemas, permitindo resolver problemas relativamente grandes com menor esforço computacional. (RAVAGNANI; CABALLERO SUÁREZ, 2012).

Alguns autores se propuseram a desenvolver algoritmos utilizando o método de programação matemática. Papoulias e Grossmann (1983) desenvolveram um modelo de programação linear (PL) com o objetivo de prever o custo mínimo de utilidades e um modelo de programação linear mista inteira (PLMI) com a finalidade de minimizar o número de unidades de troca térmica da RTC com condições fixas de operação. Floudas et al. (1986) apresentaram um modelo de PNL para gerar automaticamente as configurações da RTC com 
o mínimo custo de investimento dentre as redes que possuem o mínimo custo de utilidades e o número mínimo de unidades. Floudas et al. (1986) propuseram uma superestrutura que incorpora muitas configurações alternativas, incluindo opções como trocas em série e paralelo.

Floudas e Grossmann (1986) foram os primeiros autores que buscaram atender a necessidade de uma RTC flexível com variações pré-especificadas nas condições de operação em finitos períodos via programação matemática. Eles desenvolveram um método sequencial que atinge, além de uma rede viável, dois critérios independentes do comprimento de cada período, obtendo o mínimo custo de utilidades para cada período de operação e o mínimo número de unidades de trocadores de calor. Já o modelo apresentado por Floudas e Grossmann (1987) é uma continuação dos modelos desenvolvidos pelos próprios autores em 1986, passando a gerar automaticamente as configurações da rede multiperiódica de trocadores de calor. Eles desenvolveram um modelo de PNL, utilizando uma superestrutura que inclui muitas alternativas para um conjunto de trocas pré-estabelecidas para os diferentes períodos. Além disso, os autores propuseram uma representação gráfica com a finalidade de reduzir o tamanho do programa não linear e considerar as mudanças no ponto Pinch.

Assim, o objetivo principal deste trabalho é aperfeiçoar o algoritmo utilizado por Floudas e Grossmann (1987), buscando alcançar uma rede flexível de trocadores de calor, baseando-se nos fundamentos das técnicas de programação matemática. Propõem-se uma superestrutura diferenciada da apresentada pelos autores, além da utilização de dois conjuntos de índices e de um parâmetro para reduzir o número de variáveis e equações.

\section{MODELO}

Com a finalidade de sintetizar uma RTC flexível, utilizando o método sequencial para obter uma configuração viável da rede com o custo mínimo de investimento, mínimo número de unidades de troca térmica e mínimo custo de utilidades para cada período de operação, um procedimento sistemático é proposto. Este procedimento sugere três etapas, em que uma tem precedência sobre a outra. Na primeira etapa, deve-se resolver o modelo de PL separadamente para cada período, o qual está reportado em Floudas e Grossmann (1986) e por meio dele, são obtidas, para cada período de operação, a temperatura do Pinch e as demandas de utilidades quentes e frias. Na segunda etapa, utiliza-se das informações obtidas na primeira etapa para formular e resolver o modelo multiperiódico de PLMI, com o objetivo de encontrar o número mínimo de unidades de troca térmica. Esse modelo gera as trocas térmicas necessárias para as correntes e o calor trocado em cada unidade para cada período e está reportado em Floudas e Grossmann (1986). Na terceira etapa, utiliza-se das informações obtidas nas outras duas etapas para formular e resolver o modelo de PNL apresentado no tópico de Mínimo Custo de Investimento. Esse modelo, baseado em Floudas e Grossmann (1987), é utilizado para determinar o tamanho da unidade de troca térmica que atenda todos os períodos de operação e a configuração automática da rede.

\subsection{Mínimo Custo de Investimento}

O modelo de PNL multiperiódico apresentado neste tópico baseia-se nas superestruturas individuais desenvolvidas para cada corrente, o qual considera todas as possíveis conexões entre as trocas previstas. As capacidades térmicas, as temperaturas de entrada e saída das 
superestruturas, as unidades de troca térmica e as correntes que trocam calor em cada unidade são informações conhecidas. Para exemplificar, a Figura 1 mostra a superestrutura para o caso em que a corrente quente $\mathrm{H} 1$ troca calor com duas correntes frias $\mathrm{C} 1$ e $\mathrm{C} 2$, nas unidades $\mathrm{U} 1 \mathrm{e}$ U2.

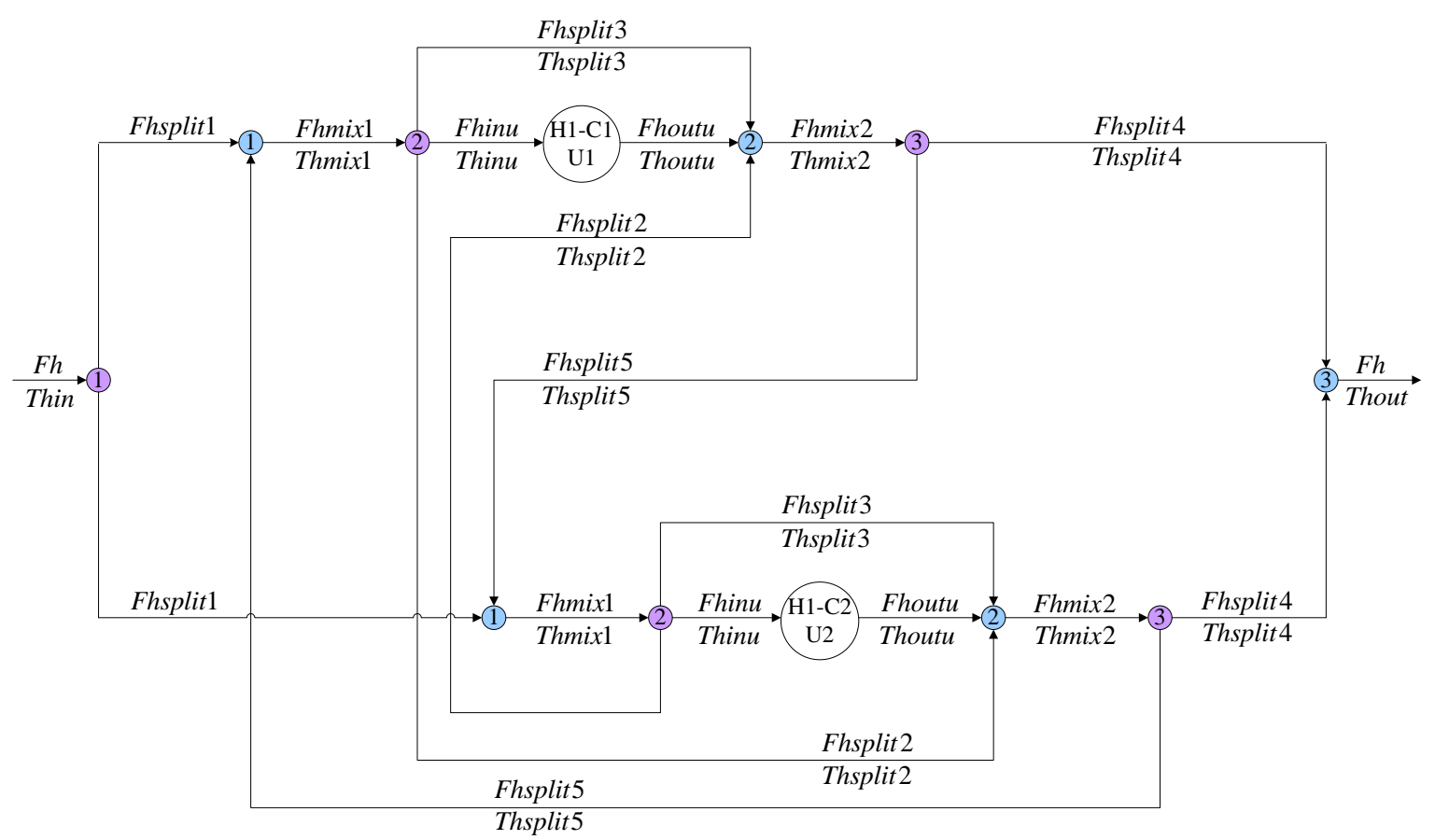

Figura 1 - Superestrutura multiperiódica para corrente quente.

As nomenclaturas apresentadas na Figura $1 \mathrm{com}$ as iniciais $F h$ e $T h$ são as capacidades térmicas e as temperaturas da corrente quente ou da utilidade quente nos ramos indicados. Essas nomenclaturas são fixas, independendo da quantidade de unidades de troca térmica que cada superestrutura possui, modificando apenas os índices de cada variável. Os círculos roxos e azuis representam os divisores e os misturadores de correntes, os quais são enumerados para auxiliar na descrição das equações do modelo. O by-pass que sai da entrada de uma unidade (divisor 2) e entra na saída de outra (misturador 2), o qual possui as nomenclaturas Fhsplit2 e Thsplit2, modifica a superestrutura adotada por Floudas e Grossmann (1987) e existe se a corrente analisada troca calor com pelo menos duas unidades.

Para as correntes e utilidades frias, as variáveis de capacidade térmica e de temperatura nos ramos recebem nomenclaturas com as iniciais $F c$ e Tc: $F \operatorname{csplit} 1_{i, j, u, t}, F c m i x 1_{i, j, u, t}$, Tcmix $_{i, j, u, t}$, Fcsplit $_{i, j, i i, u, u u, t}$, Tcsplit $_{i, j, i i, u, u u, t}$, Fcsplit $_{i, j, u, t}$, Tcsplit $_{i, j, u, t}$,

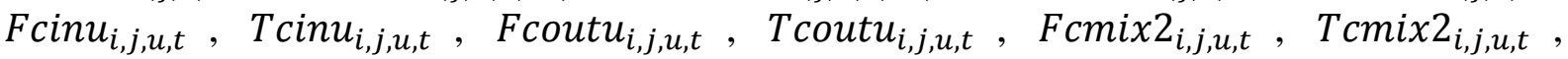
Fcsplit $_{i, j, u, t}$, Tcsplit $_{i, j, u, t}$, Fcsplit $_{i, j, i i, u, u u, t}$ e Tcsplit $5_{i, j, i i, u, u u, t}$, em que os índices $i$ ou $i i$ representam as correntes e utilidades quentes, $j$ ou $j j$ representam as correntes e utilidades frias, $u$ ou $u u$ representam as unidades de troca térmica e $t$ representa o período de operação. Para as utilidades do mesmo tipo que trocam calor com mais de uma corrente de processo, deve-se gerar superestruturas separadas para cada troca.

Outras variáveis são necessárias: $d 1_{u, t}$ e $d 2_{u, t}$ indicam a diferença de temperaturas nos 
terminais quente e frio da unidade de troca térmica; a médica logarítmica das diferenças de temperaturas, a qual é calculada pela aproximação de Chen (1987), a área da unidade de troca térmica em um período de operação, a área máxima da unidade entre os períodos e o calor trocado no equipamento são representados pelas variáveis $L M T D_{u, t}, \operatorname{Area}_{u, t}, \operatorname{Area}_{u} \mathrm{e}$ $Q u_{u, t}$; CustoTotal e CustoTotal1 indicam o custo calculado com as áreas das unidades de todos os períodos e o custo calculado com as áreas máximas de cada unidade. Os conjuntos utilizados no modelo são: $P l_{i, j}$ e $P m_{i, j}$ que indicam os pares de correntes que tem apenas uma unidade em cada sub-rede dos períodos e pares de correntes que possuem diferentes unidades na sub-rede de pelo menos um período; o par de correntes que troca calor na unidade é indicado pelo conjunto $U n_{u, i, j} ; d_{t, i, j}$ é o conjunto que indica o período dominante de um par de corrente, ou seja, período em que um par de corrente troca calor em mais de uma sub-rede do mesmo período.

A fim de reduzir o número de variáveis e de restrições envolvido no modelo, foi proposta a criação de dois conjuntos $N h_{i, u, u u, t}$ e $N c_{j, u, u u, t}$, que indicam a existência de correntes que trocam calor em unidades que estão em sub-redes sucessivas. Nesses conjuntos não se deve incluir unidades que trocam calor com as utilidades. Também foi criado um parâmetro $S u b_{u, t}$ que representa a sub-rede do período, em que a unidade está presente. Este parâmetro é definido, se a rede apresenta apenas um Pinch, pelo número 1, se a unidade de troca térmica está acima do Pinch, e pelo número 2, se a unidade está abaixo desse ponto. A partir dessas informações é possível simplificar as superestruturas das correntes, por exemplo, zerando as vazões de algumas interconexões para alcançar a troca em série entre as sub-redes para as correntes que trocam calor em mais de uma unidade, as quais estão localizadas em sub-redes sucessivas. Ressalta-se que cada unidade de troca térmica está relacionada com apenas um par de correntes e as unidades que estão na mesma sub-rede podem se interconectar de todas as maneiras.

A formulação matemática do modelo possui a função objetivo de minimizar o custo de investimento para $N$ períodos de operação. Algumas restrições são apresentadas apenas para as correntes quentes, porém as mesmas devem ser geradas para as correntes frias:

$\min$ Custo $=$ CustoTotal

Sujeito a:

- Balanço de massa nos divisores inicial (1), (2) e (3) para as correntes quentes e frias: a capacidade térmica que entra no divisor deve ser igual à somatória da capacidade térmica que sai do mesmo divisor.

- Igualdade de temperatura nos divisores (2) e (3) para as correntes quentes e frias: a temperatura que entra no divisor deve ser igual à temperatura de todos os ramos que saem do mesmo divisor.

- Balanço de massa nos misturadores (1), (2) e (3) para as correntes quentes e frias: o somatório das capacidades térmicas que entram no misturador deve ser igual à capacidade térmica que sai do mesmo misturador. 
- Balanço de energia nos misturadores (1), (2) e (3) para as correntes quentes e frias: o somatório das capacidades térmicas multiplicadas pelas temperaturas correspondentes que entra no misturador deve ser igual a capacidade térmica multiplicada pela temperatura correspondente que sai do mesmo misturador.

- Viabilidade das temperaturas: as correntes, que trocam calor em mais de uma unidade e estas unidades estão em sub-redes diferentes, devem apresentar uma temperatura de saída maior ou igual no trocador de calor que está na sub-rede superior.

- Viabilidade das capacidades térmicas:

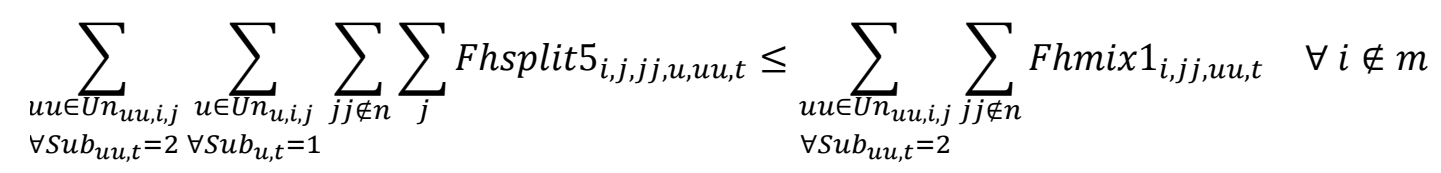

- Aproximação mínima das temperaturas:

$$
d 1_{u, t} \geq \Delta T_{\min } \text { e } d 2_{u, t} \geq \Delta T_{\min }
$$

- Balanço de massa e de energia em cada unidade de troca térmica:

$$
\begin{aligned}
& \text { Fhinu }_{i, j, u, t}=\text { Fhoutu }_{i, j, u, t} \quad \forall u \in U n_{u, i, j} \\
& Q u_{u, t}=\text { Fhinu }_{i, j, u, t} \cdot\left(\text { Thinu }_{i, j, u, t}-\text { Thoutu }_{i, j, u, t}\right) \quad \forall u \in U n_{u, i, j}
\end{aligned}
$$

Em que $Q u_{u, t}$ será igual a carga de calor obtida pelo modelo de PLMI para os pares que pertencem ao conjunto $P l_{i, j}$ e $P m_{i, j}$ em que o período analisado é o dominante. Para os períodos não dominantes dos pares que pertencem ao conjunto $P m_{i, j}$, a carga de calor obtida pelo modelo de PLMI será igual ao somatório de $Q u_{u, t}$ em todas as unidades que possuem o par de corrente analisado.

- Projeto dos trocadores de calor:

$$
\begin{aligned}
& d 1_{u, t}=\text { Thinu }_{i, j, u, t}-\text { Tcoutu }_{i, j, u, t} \quad \forall u \in U n_{u, i, j} \\
& d 2_{u, t}=\text { Thoutu }_{i, j, u, t}-\text { Tcinu }_{i, j, u, t} \quad \forall u \in U n_{u, i, j} \\
& Q u_{u, t}=\operatorname{Co}_{u} \cdot \text { Area }_{u, t} \cdot \operatorname{LMTD}_{u, t} \\
& \text { CustoTotal }=\sum_{u} \sum_{t}\left(a+b \cdot \text { Area }_{u, t}{ }^{c}\right)
\end{aligned}
$$

Em que $C o_{u}$ é o coeficiente global de transferência de calor na unidade e $a, b, c$ são os coeficientes da equação do custo. O custo de investimento real é calculado pela Equação 10:

$$
\text { CustoTotal1 }=\sum_{u}\left(a+b \cdot \operatorname{AreaM}_{u}{ }^{c}\right)
$$

Segundo Floudas et al. (1986), o aumento de correntes recicláveis (Fhsplit5 $5_{i, j, j j, u, u u, t} \mathrm{e}$ 
Fcsplit $_{i, j, i i, u, u u, t}$ ) implica no decréscimo da média logarítmica das diferenças de temperaturas, resultando no aumento da área das unidades e, consequentemente, no aumento do custo de investimento da rede. Com base nesta afirmação, este modelo busca otimizar a área de todas as unidades em todos os períodos de operação, visando diminuir o número de correntes recicláveis em todos os períodos e não apenas diminuir esse número no período em que a unidade possui área máxima. Assim, com esse modelo é possível obter a configuração da rede, a qual é definida simplesmente pelas capacidades térmicas não negativas que indicam as interconexões das correntes requeridas para as unidades de troca térmica.

\section{ESTUDO DE CASO}

O exemplo apresentado neste tópico foi retirado de Floudas e Grossmann (1987). Este problema é composto por duas correntes quentes, duas correntes frias, uma utilidade quente (vapor $-S$ ) e uma utilidade fria (água de resfriamento - $W$ ), operando em três períodos de operação. Os valores das capacidades térmicas e das temperaturas de entrada e de saída das correntes sofrem alterações de um período para o outro e são mostrados na Tabela 1.

Tabela 1 - Dados de entrada

\begin{tabular}{ccccccccccc}
\hline & \multicolumn{3}{c}{ Período 1 } & \multicolumn{3}{c}{ Período 2 } & \multicolumn{3}{c}{ Período 3 } \\
\cline { 2 - 10 } Corrente & Tin & Tout & $F$ & Tin & Tout & $F$ & Tin & Tout & $F$ \\
& $\left({ }^{\circ} \mathrm{C}\right)$ & $\left({ }^{\circ} \mathrm{C}\right)$ & $\left(\mathrm{kW} /{ }^{\circ} \mathrm{C}\right)$ & $\left({ }^{\circ} \mathrm{C}\right)$ & $\left({ }^{\circ} \mathrm{C}\right)$ & $\left(\mathrm{kW} /{ }^{\circ} \mathrm{C}\right)$ & $\left({ }^{\circ} \mathrm{C}\right)$ & $\left({ }^{\circ} \mathrm{C}\right)$ & $\left(\mathrm{kW} /{ }^{\circ} \mathrm{C}\right)$ \\
\cline { 2 - 11 } $\mathrm{H} 1$ & 249 & 100 & 10,55 & 229 & 120 & 7,032 & 249 & 100 & 10,55 \\
$\mathrm{H} 2$ & 259 & 128 & 12,66 & 239 & 148 & 8,44 & 259 & 128 & 12,66 \\
$\mathrm{C} 1$ & 96 & 170 & 9,144 & 96 & 170 & 9,144 & 116 & 150 & 6,096 \\
$\mathrm{C} 2$ & 106 & 270 & 15 & 106 & 270 & 15 & 126 & 250 & 10 \\
\hline
\end{tabular}

Os modelos foram resolvidos utilizando-se a ferramenta de otimização GAMS, com a versão $22.6 \mathrm{em}$ um processador Intel Core 5, 2.60 GHz. Para os dois primeiros passos do método sequencial, utilizou-se os modelos de PL e PLMI propostos por Floudas e Grossmann (1986) e $10^{\circ} \mathrm{C}$ para a temperatura mínima de aproximação. Esses modelos foram resolvidos por meio do solver CPLEX em um tempo de processamento menor que 1 segundo, atingindo as mesmas quantidades de utilidade quente e fria, os mesmos pontos Pinch e as mesmas unidades para configuração da RTC que os autores apresentaram, porém com mudança na quantidade de calor trocada em cada uma das unidades. Conforme Ravagnani e Caballero Suárez (2012), pode existir soluções com diferentes configurações que alcançam os mínimos custos de utilidades e o número mínimo de trocadores de calor. A Tabela 2 mostra os pares de correntes em cada trocador de calor e a quantidade de calor trocada em cada unidade de troca térmica e em cada período.

Para o terceiro passo do método, deve-se aplicar o algoritmo desenvolvido neste trabalho. Assim, com as informações encontradas nos passos anteriores, é possível identificar que o parâmetro $S u b_{u, t}$ recebe: o valor $1 \mathrm{em}$ todas as unidades do período 2, pois o mesmo não possui sub-rede; o valor 1 nos períodos 1 e 3 para a unidade $U 1$; o valor 2 nos períodos 1 e 3 para as unidades $U 3, U 4, U 5, U 6$ e $U 7$; e para a unidade $U 2$, o valor é 1 para o período 1 e o valor é 2 para o período 3. Observa-se que a utilidade fria necessita ser renomeada para $W 1$ e $W 2$, pois a mesma é usada tanto na unidade 6 quanto na unidade 7. Os pares de correntes 
são divididos em dois conjuntos: $P l$ é formado pelos pares $H 1-C 1, H 1-C 2, H 1-W 1, H 2$ $W 2$ e $S$ - $C 2$; e $P m$ é formado pelo par $H 2$ - $C 2$ em que o período 1 é o período dominante. O conjunto $N h$ é composto pelo quarteto $H 2$ - U2 - U5 - T1 e o conjunto $N c$ é composto pelos quartetos: $C 2$ - $U 2$ - $U 4$ - T1 e $C 2$ - U2 - U5 - T1.

Tabela 2 - Trocas e quantidades de calor $(k W)$ em cada período

\begin{tabular}{ccccc}
\hline Unidade de troca térmica & Troca & Período 1 & Período 2 & Período 3 \\
\hline 1 & S-C2 & 338,4 & 1602,128 & $10{ }^{*}$ \\
2 & H2-C2 & 126,6 & 0 & 0 \\
\cline { 5 - 6 } 3 & H1-C1 & 676,656 & 676,656 & 207,264 \\
4 & H1-C2 & 817,934 & 89,832 & 1045,846 \\
5 & H2-C2 & 1177,066 & 768,04 & 184,154 \\
6 & H1-W & 77,36 & 0 & 318,84 \\
7 & H2-W & 354,794 & 0 & 1474,306 \\
\hline
\end{tabular}

* Linha que indica a divisão entre sub-redes

Baseado em Floudas e Grossmann (1987), utiliza-se $0,1^{\circ} \mathrm{C}$ para a temperatura mínima de aproximação e para os coeficientes globais de transferência de calor e os dados de custo, utiliza-se os valores apresentados na Tabela 3, a qual possui também a área das unidades de troca térmica encontradas com o desenvolvimento do modelo de PNL. Esse modelo é resolvido por meio do solver CONOPT3, o qual utiliza o método do gradiente reduzido (GAMS, 2008), com um tempo de processamento menor que 1 segundo.

Tabela 3 - Coeficientes de transferência de calor e área das unidades

\begin{tabular}{|c|c|c|c|}
\hline Unidade & Troca & $\mathrm{Co}_{u}\left(\mathrm{~kW} /\left(\mathrm{m}^{2} \cdot{ }^{\circ} \mathrm{C}\right)\right)$ & $\operatorname{AreaM}\left(\mathrm{m}^{2}\right)$ \\
\hline 1 & S-C2 & 0,8 & 28,756 \\
\hline 2 & $\mathrm{H} 2-\mathrm{C} 2$ & 1 & 11,765 \\
\hline 3 & $\mathrm{H} 1-\mathrm{C} 1$ & 1 & 28,155 \\
\hline 4 & $\mathrm{H} 1-\mathrm{C} 2$ & 1 & 84,995 \\
\hline 5 & $\mathrm{H} 2-\mathrm{C} 2$ & 1 & 57,992 \\
\hline 6 & H1-W & 0,4 & 10,629 \\
\hline 7 & $\mathrm{H} 2-\mathrm{W}$ & 0,3 & 34,923 \\
\hline
\end{tabular}

A rede proposta neste trabalho apresenta os mesmos custos de utilidades atingidos por Floudas e Grossmann (1987), porém com custo de investimento menor, conforme mostra a Tabela 4 e com menos by-passes, o que diminui ainda mais os gastos na implantação da rede. A configuração da rede é definida pelas capacidades térmicas não negativas que indicam as interconexões das correntes nas unidades de troca térmica.

Dessa forma, pode-se concluir que o resultado atingido por Floudas e Grossmann (1987) é um ótimo local e a redução dos gastos ocorre devido à melhoria realizada na superestrutura e no algoritmo do modelo de PNL. Não é possível concluir se o resultado 
atingido neste trabalho é um ótimo global ou um ótimo local, pois o solver adotado não utiliza um método de otimização global.

Tabela 4 - Comparação dos custos

\begin{tabular}{lcccc}
\hline & \multicolumn{3}{c}{ Custo com utilidades (\$) } & Custo de \\
& Período 1 & Período 2 & Período 3 & investimento \\
& $\$$ \$) \\
\hline Floudas e Grossmann (1987) & $8,418 / \mathrm{h}$ & $27,465 / \mathrm{h}$ & $11,033 / \mathrm{h}$ & $269.380,00$ \\
Presente trabalho & $8,418 / \mathrm{h}$ & $27,465 / \mathrm{h}$ & $11,033 / \mathrm{h}$ & $249.866,75$ \\
\hline
\end{tabular}

\section{CONCLUSÕES}

Redes com condições de operação fixas, muitas vezes, não atendem eficientemente as solicitações das indústrias, sendo necessário gerar redes flexíveis que suportam mudanças nas condições de operação em um número finito de períodos de operação. Este trabalho apresentou um modelo de programação matemática, para alcançar a configuração da rede flexível automaticamente com o custo mínimo de investimento. Propôs uma melhoria na superestrutura multiperiódica e no algoritmo do modelo de PNL utilizado por Floudas e Grossmann (1987). O estudo de caso realizado resultou em redes com custos finais menores do que os custos apresentados por Floudas e Grossmann (1987), comprovando a viabilidade do modelo desenvolvido neste trabalho.

\section{REFERÊNCIAS}

Chen, J. J. J. Letters to the editors: comments on improvements on a replacement for the logarithmic mean. Chem. Eng. Sci., v. 42, n.10, p. 2488-2489, 1987.

Floudas, C. A.; Ciric, A. R.; Grossmann, I. E. Authomatic synthesis of optimum heat exchanger network configurations. AIChE J., v. 32, n. 2, p. 276-290, 1986.

Floudas, C. A.; Grossmann, I. E. Synthesis of flexible heat exchanger networks for multiperiod operation. Comput. Chem. Eng., v. 10, n. 2, p. 153-168, 1986.

Floudas, C. A.; Grossmann, I. E. Automatic generation of multiperiod heat exchanger network configuration. Comput. Chem. Eng., v. 11, n. 2, p. 123-142, 1987.

GAMS Development Corporation. GAMS - The Solver Manuals. Washington: 2008.

Módenes, A. N. Síntese de Redes de Trocadores de Calor Flexíveis. 1995. 100 f. Dissertação (Mestrado em Engenharia Química) - Curso de pós-graduação em engenharia química, Universidade Estadual de Maringá, Maringá, 1995.

Papoulias, S. A.; Grossmann, I. E. A structural optimization approach in process synthesis. Part II: Heat recovery networks. Comput. Chem. Eng., v. 7, n. 6, p. 707-721, 1983.

Ravagnani, M. A. S. S.; Caballero Suárez, J. A. Redes de Cambiadores de Calor. Alicante: Editora de la Universidad de Alicante, 2012. 